\title{
Serial block face scanning electron microscopy in cell biology: Applications and technology
}

DOI:

10.1016/j.tice.2018.08.011

\section{Document Version}

Accepted author manuscript

Link to publication record in Manchester Research Explorer

\section{Citation for published version (APA):}

Smith, D., \& Starborg, T. (2018). Serial block face scanning electron microscopy in cell biology: Applications and technology. Tissue and Cell. https://doi.org/10.1016/j.tice.2018.08.011

\section{Published in:}

Tissue and Cell

\section{Citing this paper}

Please note that where the full-text provided on Manchester Research Explorer is the Author Accepted Manuscript or Proof version this may differ from the final Published version. If citing, it is advised that you check and use the publisher's definitive version.

\section{General rights}

Copyright and moral rights for the publications made accessible in the Research Explorer are retained by the authors and/or other copyright owners and it is a condition of accessing publications that users recognise and abide by the legal requirements associated with these rights.

\section{Takedown policy}

If you believe that this document breaches copyright please refer to the University of Manchester's Takedown Procedures [http://man.ac.uk/04Y6Bo] or contact uml.scholarlycommunications@manchester.ac.uk providing relevant details, so we can investigate your claim.

\section{OPEN ACCESS}




\title{
Serial Block Face Scanning Electron Microscopy in Cell Biology: Applications and Technology
}

\author{
David Smith* and Tobias Starborg \\ Wellcome Trust Centre for Cell-Matrix Research, \\ Faculty of Biology, Medicine and Health, \\ University of Manchester, \\ Michael Smith Building, \\ Oxford Road, \\ Manchester M13 9PT \\ UNITED KINGDOM
}

*To whom correspondence should be addressed:

David Smith (david.smith-5@manchester.ac.uk)

Running Header: SBFSEM in Cell Biology 


\section{Abstract}

Three-dimensional electron microscopy (3DEM) is an imaging field containing several powerful modalities such as serial section transmission electron microscopy and electron tomography. However, large-scale 3D studies of biological ultrastructure on a cellular scale have historically been hampered by the difficulty of available techniques. Serial block face scanning electron microscopy (SBFSEM) is a 3DEM technique, developed in 2004, which has greatly increased the reliability, availability and throughput of 3DEM. SBFSEM allows for 3D imaging at resolutions high enough to resolve membranes and small vesicles whilst having the capability to collect data with a large field of view. Since its introduction it has become a major tool for ultrastructural investigation and has been applied in the study of many biological fields, such as connectomics, cellular and matrix biology. In this review, we will discuss biological SBFSEM from a technical standpoint, with a focus on cellular applications and also subsequent image analysis techniques.

\section{Keywords}

Serial block face scanning electron microscopy, SBFSEM, SBF-SEM, SBEM. 


\section{Introduction}

Although the earliest discoveries regarding the cell and its ultrastructure were made using light microscopy, the advent of electron microscopy (EM) and the development of suitable sample preparation techniques made it possible to examine the cellular ultrastructure in finer detail (figure 1). Standard transmission electron microscopy (TEM) of plastic embedded cells has enabled researchers to examine cellular ultrastructure at a resolution suitable to trace individual phospholipid bilayers (Glauert, 1984).The increase in resolution allows researchers to examine cell-cell and cellmatrix interactions that would be impossible to resolve using photons of light. TEM can excel in delivering high-resolution images of exceedingly thin (20-70nm) slices of tissue, and various techniques such as serial section TEM and electron tomography exist that allow this high-resolution approach to extend into three-dimensions. Electron microscopists have become proficient in understanding how to interpret the two-dimensional slices as part of larger volumes of tissue, and take advantage of these 3D electron microscopy (3DEM) techniques to produce truly 3D datasets. 3D TEM in its various forms, despite its continued power and importance, has limitations, with high costs, difficulty and unreliability being chief among them. Over the past 30 years several 3DEM techniques have been developed that are based on the scanning electron microscope (SEM). These techniques sacrifice the ultra-high resolution of the TEM in exchange for faster, easier and more reliable data collection. They are focused ion beam scanning electron microscopy (FIBSEM), array tomography and the focus of this review: serial block face scanning electron microscopy (SBFSEM). In order to illustrate the advantages and disadvantages of the 3DEM techniques as well as throw some light on how the field has developed over time, we will briefly run through each technique below. 


\subsection{Stereo pairs}

The simplest of the 3D TEM techniques is to generate a stereo pair where two images are taken at slightly different tilt angles to mimic the separation of human eyeballs (Turner, 1981). A tilt pair can be taken on a standard TEM. The tilt pair is effectively two angled 2D projections of planes within the 3D sample volume, which allows users to 'see' within the slice. If a thicker section is tilted it is possible to see how the structures within the section interact, but it is difficult to interrogate the images in a scientific manor. Although many techniques have been developed to quantify internal structures (Ghosh, 1981), stereo images are more suited to demonstrating concepts than to facilitating data analysis.

\subsection{Transmission electron tomography}

Taking the concept of tilting the sample one stage further, electron tomography is a technique in which numerous images are taken of individual tissue sections which are tilted at regular angular intervals. Each image is a projection of all of the material within the section. By back projecting the images, it is possible to generate a 3dimensional reconstruction of the tissue in the section. The electron tomogram produces a high-resolution (typically down to a few nanometers) reconstruction of the sample, but the field of view is normally only a few micrometers (Barcena and Koster, 2009). The technique is best used for examining small regions at high resolution however it is possible to combine low-resolution electron tomography with serial sectioning if the examination of larger volumes is desired. By imaging serial thick sections (1-2 $\mu \mathrm{m}$ per section), large volumes of tissue can be examined using a 400kV microscope (Soto et al., 1994). Before we purchased our serial block face 
equipment we examined embryonic tendon development using stacks of sixty $300 \mathrm{~nm}$ thick tomograms (Kalson et al., 2013). We would not recommend this approach, however, as it is very time consuming and has a high risk of failure, although the technique does have the advantage that it allows for specific regions to be revisited at higher resolution.

\subsection{Serial Section TEM}

Standard serial section TEM (SsTEM) involves collecting a series of $50-70 \mathrm{~nm}$ sections that span the volume of interest and imaging each one separately (Rieder, 1981). The process requires a high level of technical skill, as the resin embedded block has to be trimmed perfectly in order for the sections to leave the block as a single ribbon. There are many opportunities to lose or damage slices during the procedure and even experienced microscopists can make mistakes when sectioning. In the context of SSTEM this means that the whole process must be restarted, as otherwise the resulting dataset would be incomplete. In the early days of SSTEM the images were collected onto negatives and to create a 3D model the negatives could be enlarged and traced in order to cut out the cellular shape in expanded polystyrene sheets (Pedler and Tilly, 1966). Fortunately in the present era of high-powered computing, images can be aligned and volumes can be generated with much greater ease (Fiala, 2005; Kremer et al., 1996). Despite its high difficulty, ssTEM is still in use today (Fischer et al., 2018; Tsukamoto and Omi, 2017) and has the distinct advantages of giving higher resolution when compared to the block face imaging techniques and also providing for the ability to re-image archived slices. 


\subsection{Array tomography}

An alternative to collecting serial TEM sections is to use a technique that has been termed array tomography (Micheva and Smith, 2007). Here serial sections of a sample that has been stained en-bloc with heavy metals are collected onto a substrate and imaged in an SEM. The development of array tomography has been greatly helped by automated methods of collecting the serial sections, such as the Automatic Tape-collecting Ultra-Microtome (Schalek et al., 2011). The Automatic Tape-collecting Ultra-Microtome is a device comprising of an ultramicrotome, which automatically collects sections of resin-embedded tissue on Kapton tape in long reels. The tape is then cut into sections and attached to silicon wafers for imaging in the SEM. The advantage of this technique is the reduced amount of manual manipulation, which reduces the loss of, or damage to individual sections. The sample can also be archived and revisited. Software is being developed specifically to image these arrays of sections, speeding up the data acquisition process considerably (Hayworth et al., 2014). The sample collection still appears to be time consuming and requires a highly trained user who is capable of reproducibly preparing high quality TEM sections, however the technique does exceed in its versatility, facilitating repeated imaging at different resolutions and reducing the problem of sample charging encountered with the block face imaging techniques (Wacker et al., 2018).

\subsection{Block Face Imaging techniques}

The block face imaging techniques consist of focussed ion beam SEM (FIBSEM) and, the focus of this review, serial block face SEM (SBFSEM). In contrast with the previously mentioned techniques, which involve imaging thin slices of stained 
material cut from an epoxy resin block, the FIB-SEM and SBFSEM directly image the sample block face.

\subsubsection{Focussed-Ion beam scanning electron microscopy}

Focussed-ion beam SEM takes advantage of the dual beam instruments sold by many of the microscope manufacturers. The instruments were initially developed for materials science applications but are now frequently being used for volume imaging of biological samples (Knott et al., 2008; Xu et al., 2017). The basic instrument has an electron column, which can be used for standard scanning electron microscopy. At an angle to the electron column is an ion column, which generates positively charged ions (frequently gallium) and accelerates these towards the sample in a controlled manner. Both the electron column and the ion column have scan coils, which allow the beams to be accurately moved across the surface of the sample. As the ion beam is scanned over the sample, high-momentum ions mill away material in a highly controlled fashion (Volkert and Minor, 2007). Images are taken between rounds of milling to remove a thin layer, generating a series of images that constitute a three-dimensional volume. The great precision with which the ion beam can be moved across the sample allows for arbitrarily thin slices between images meaning that datasets can have higher Z-resolutions than SSTEM and SBFSEM. Users have also found that they are able to image samples that have been prepared for conventional TEM using FIBSEM due to the fact that they can use long dwell times to compensate for the relatively low contrast (Knott et al., 2008). The disadvantage of FIBSEM is that as the area to be milled increases the time for milling increases, which affects the overall data collection rate. The process also requires that the microscope be re-focussed every time a slice is taken, which increases the chance of imaging problems during a run (Xu et al., 2017). 


\subsubsection{Serial Block Face scanning electron microscopy}

Serial block face scanning electron microscopy is known by many abbreviations, including SBEM and SBSEM, but for the purpose of this review we will use the term SBFSEM. The basic SBFSEM machine uses a microtome that sits within the chamber of an SEM to remove thin slices of the embedded sample (figure 2). The top of the block face is imaged before the sample is cut at a specified depth by the microtome, revealing a new block face, which is again imaged and the process repeated. The first example of a microtome that fit within an SEM was described by Leighton in 1981 (Leighton, 1981). Leighton built a fully working microtome that was able to cut a thin section of resin which could be imaged in the SEM. However the technique was limited at the time, both because the non-conductive samples had to be carbon coated in between sectioning to reduce charging (Biazik et al., 2015) and because computers at the time lacked the power required to work with the large numbers of digital images that could be generated (for context: in 1981 Microsoft released MS-DOS, their first operating system). Denk and Horstmann revisited the concept years later and fit their microtome inside an SEM with a variable pressure chamber that could introduce a small amount of water vapour into the system (Denk and Horstmann, 2004). Adding water vapour reduced sample charging during imaging, allowing the visualisation of non-conductive samples without the need for coating between slices. The Denk and Horstmann microtome was eventually commercialised to become the 3view ${ }^{\mathrm{TM}}$ (Gatan, UK). The 3view ${ }^{\mathrm{TM}}$ is designed to replace the standard door of any donor SEM that can fit the microtome under the beam. As the benefits of in chamber microtoming have become more apparent other manufactures have developed competing systems. In 2014 FEI (now Thermo Fisher Scientific) announced the Teneo Volumescope ${ }^{\text {TM }}$ (Korkmaz, 2016). The Volumescope is still relatively new in the field however it has been utilised in several 
recent studies (Ghosh et al., 2018; Hussain et al., 2018; Parkyn Schneider et al., 2017; Wang et al., 2018).

SBFSEM has several advantages over other 3DEM techniques. Firstly, compared to FIBSEM (the milling process of which can be very time consuming), the process of cutting the sample using the in-chamber microtome between images takes only seconds. As a result the primary factor in determining how long it takes to collect a dataset is simply how long it takes to scan each individual pixel for each image, making runs quicker. The knife can also quickly remove a greater volume of material than the ion beam of the FIBSEM, meaning that larger volumes of tissue can feasibly be scanned (figure 3). Both techniques can reach a minimum $X, Y$ pixel size of around 3-5nm (Daum et al., 2017; Feeney et al., 2018) however voxel size in the Zdirection is at best 20nm in SBFSEM (Chen et al., 2017) (a limit set by the cutting of the knife) whereas FIBSEM allows the milling of arbitrarily thin sections. Compared to SSTEM, both FIBSEM and SBFSEM sacrifice ultimate resolution in exchange for greater ease of use and reliability: both block face techniques allow the user to leave the microscope on 'autopilot' once a run has been initiated (assuming there are no problems encountered with cutting/milling or imaging), and thus the total work hours needed to produce a complete dataset are vastly reduced. Techniques can be combined to ascertain information at different resolutions, for instance electron tomography is highly complementary to SBFSEM. It is possible to image the same sample using SBFSEM along with electron tomography in order to get information across many length scales (Godwin et al., 2017). Problems with the technique include the fact that that the resin-embedded block is irradiated with damaging highenergy electrons, leading to imaging issues and problems with knife cutting. The process is also inherently destructive, and as mentioned earlier the slice thickness is limited to 20nm, which limits the ultimate Z-resolution. For an overview and 
comparison of the aforementioned techniques see (Peddie and Collinson, 2014; Webb and Schieber, 2018).

SBFSEM has been used to examine the structure of cells and tissue at many length scales. At the larger end of the scale the technique was used to image and model the entire silk spinneret system, including glands, nerves and other tissues, of a tanaid crustacean (Kaji et al., 2016). Structures which are dozens of microns in length such as axons (Kornfeld et al., 2017) and entire collagen fibrils (Svensson et al., 2017) can be imaged as well as large populations of cells in one automated run (Pollreisz et al., 2018; Salo et al., 2018). At the level of whole cells, SFBFEM has been used to image whole glomerular podocytes (Lausecker et al., 2018) and parasitic T. brucei cells (Hughes et al., 2017). On the subcellular level, studies have utilised SBFSEM to examine the ultrastructure of many cell components. A recent study examined the morphology of carnivorous plant nuclei in order to examine novel membrane extensions (Plachno et al., 2017). Sub-nuclear structures such as individual chromosomes (Chen et al., 2017) and the mitotic spindle (Nixon et al., 2017) have been imaged and modelled. At the nanometer scale, SBFSEM is able to resolve structures such as vesicles (Chuang et al., 2015), T-tubules (Pinali et al., 2013) and the cristae of mitochondria (Vincent et al., 2016). These examples are not exhaustive and rather are provided to show a broad cross-section of recent research and illustrate that SBFSEM is a versatile and in-demand technique. Advances in technology, whether they are specific to SBFSEM or more broadly effective, will mean that the capabilities of this technique will only increase over time, and the boundaries of what is possible both at the small and large ends of the scale are being pushed constantly. 


\section{Technical Procedures of SBFSEM}

There are several stages of sample preparation and setup that take an SBFSEM sample from an area of living tissue to a set of voxel intensity values stored on a computer. The most common sample preparation involves immersion in various liquid fixatives and stains. Firstly, the tissue must be fixed in an aqueous solution of formaldehyde and glutaraldehyde. To ensure adequate fixation and stain penetration, the tissue must be in sufficiently small pieces ( $1 \mathrm{~mm}$ cubes). Several steps of heavy metal stains are then performed to enhance the contrast of the tissue when viewed in the electron microscope. The sample is then embedded in resin which is cured before the block is further shaped and polished in order to fit in the chamber of the microscope and aid in the alignment of the in-chamber ultramicrotome. Imaging settings are selected and a 'run' can begin. After a successful run, the collection of images is processed computationally as desired and a 3D dataset is produced. Subsequently, data analysis and image segmentation is performed. This review shall break down important parts of this process and describe particular areas of challenge, deviation of technique and innovation.

\subsection{Sample preparation}

In a standard TEM preparation an en-bloc osmium stained sample can be stained with uranyl acetate and lead citrate (Reynolds, 1963) after sectioning in order to enhance contrast. However this is not possible within the vacuum of the SEM chamber and thus for SBFSEM the sample must be contrasted before imaging begins. Modern protocols are based on a procedure developed by Deerinck (Deerinck et al., 2010b). The protocol is based on the rOTO stains developed in the 1980s (Willingham and Rutherford, 1984). Post-fixation, multiple en-bloc heavy metal stains - osmium tetroxide, uranyl acetate and lead aspartate - are used to 
greatly increase the metal concentration within the tissue providing contrast. The first, of two, osmium incubations uses reduced osmium, which encourages more membrane/lipid staining. Osmium will mainly bind to $\mathrm{C}=\mathrm{C}$ bonds in unsaturated fats (Khan et al., 1961), providing a strong membrane stain. Subsequently adding the mordant thiocarbohydrazide, which binds the osmium left by the first incubation, allows the binding of further osmium in a subsequent incubation therefore enhancing the stain (Seligman et al., 1966). The uranium and lead salts both bind a range of cellular components with different affinities and further enhance the overall contrast.

The original 'Deerinck' protocol is a good starting point for SBFSEM sample processing, however it works best for lipid rich tissues such as brain. In other tissues groups have been experimenting by tweaking the protocol in order to optimise staining for specific biological materials. For example at Manchester we have changed the mordant to tannic acid in order to enhance staining of samples rich in extra-cellular matrix components (Starborg et al., 2013). The use of uranyl acetate in EM is becoming increasingly difficult as governments and research laboratories have moved to introduce stricter regulations on the use of radioactive materials. Work has examined replacing the uranyl acetate used in SBFSEM with lanthanides in order to retain contrast whilst avoiding having to deal with these regulations and it was reported that the use of the lanthanides samarium and gadolinium generally improved contrast and reduced sample charging (Odriozola et al., 2017). The requirement for large amounts of heavy metal staining has made groups re-visit stains that were developed during the high voltage period of EM, when people experimented using mega electron volt (MeV) microscopes (Glauert, 1979). Chris Hawes (Oxford Brookes, UK) has successfully imaged archived root tip samples that had been prepared with very long incubations in Zinc iodide (Kittelmann et al., 2016). 
Current staining protocols generally work well with most samples, but they are not perfect. Three general problems with current protocols are: stain penetration, the fact that the protocols take a long time (generally a week), and imperfect initial fixation. For small samples the stain penetration may not be an issue, but for groups wanting to stain whole brains there is a need for thorough, even staining. By delaying the reduction of the osmium (Hua et al., 2015), or by using a smaller mordant such as pyrogalol (a small precursor of tannic acid) (Mikula and Denk, 2015) the stain penetration increases from a few hundred micrometers to several millimeters. Regarding the time needed for sample processing, most of the current embedding and staining protocols take nearly a week because of the large number of long incubations needed. Webb and Schieber have been working to increase the speed of staining and embedding by using microwave energy to speed up the staining steps (Webb and Schieber, 2018). Through the use of low energy microwaves they are able to embed the sample with an SBFSEM-suitable heavy metal stain within a single day.

Finally regarding fixation; currently, SBFSEM samples are fixed chemically using a mixture of aldehydes, a process which may take several minutes depending on the rate of infiltration. Aldehyde fixation is well known for causing changes in cell morphology (Mazzone et al., 1980; Schultz et al., 1957; Szczesny et al., 1996) and for allowing enzymatic processes to continue whilst the fixatives penetrate the tissue (Kellenberger et al., 1992). Cryofixation is advantageous in this respect as when done correctly it halts cellular processes and preserves tissue ultrastructure (Saga, 2005). Standard cryofixation protocols are unsuitable for SBFSEM as the resultant heavy metal staining levels are sub-optimal to provide contrast. There have been several studies which examine ways to modify cryofixation protocols to rectify this. 
Webb and Schieber have shown that it is possible to adapt the Deerinck protocol to work in acetone and methanol following high pressure freezing (Webb and Webb, 2015; Webb and Schieber, 2018), and another recently developed technique, the CryoChem method (CCM), utilises the rehydration of cryofixed samples, allowing enbloc staining which is suitable for SBFSEM (Tsang et al., 2018). CCM also facilitates diaminobenzidine (DAB) staining (previously impossible with cryofixation techniques and discussed subsequently in section Targeted staining) and enhances the ability to perform correlative light-electron microscopy (CLEM) on cryofixed samples (CLEM is discussed in section Correlative SBFSEM). It utilises a protocol that combines cryo- and chemical fixation by using a mixture of chemical fixatives during freezesubstitution following cryofixation. The sample is then gradually rehydrated using a series of acetone solutions suitable for en-bloc staining, DAB labelling and fluorescence microscopy. Despite limitations (such as the potential for damage due to ice crystal formation and restrictions on sample size) cryofixation techniques show promise for expanding the capabilities of block face electron microscopy.

\subsection{Targeted staining}

Standard staining techniques for SBFSEM, like most EM techniques, produce contrast based on the relatively non-specific binding of heavy metal salts. These methods excel when imaging large areas of a sample at high resolution, however if the intention is to locate a specific protein of interest then the fact that all cellular components are stained can make life difficult. Within the EM field there are a variety of techniques that can be used in order to localise proteins of interest, however SBFSEM cannot utilise any technique where specific staining occurs after the section has been taken (Biazik et al., 2015). In order to localise proteins with more accuracy en-bloc immunological labelling with nano-gold particles can be performed before the staining process begins (Robinson et al., 2000). The epitope 
of interest is targeted with a primary antibody and the secondary antibody is labelled with a small $(1.4 \mathrm{~nm})$ gold particle. The gold particle's small size reduces the need for significant membrane disruption, which would be seen as gross damage within the electron microscope. However the gold particle needs enlarging via silver enhancement before it can be visualised by SBFSEM (Biazik et al., 2015). DNA can be selectively stained using the NAMA-Ur method which utilises the extraction of RNA and phosphoprotein phosphate groups as well as the blocking of carboxyl and amino groups to ensure that applied uranyl can only bind DNA (Testillano et al., 1991). This method was successfully used in the interrogation of nucleic chromatin architecture using SBFSEM (Rouquette et al., 2009).

A number of localisation techniques take advantage of the precipitation of DAB. Horseradish peroxidase (HRP) can be attached to the target (for instance via immunohistochemical staining or genetic modification) before the sample is incubated with $\mathrm{DAB}$ and hydrogen peroxide. The DAB is then precipitated in the presence of the peroxidase. A brown precipitate is formed which is visible under the light microscope and can be seen by EM following staining as the precipitate binds osmium (Hanker, 1979). HRP has been used in EM for many years and has been successfully used in serial block face imaging to visualise specific regions of cells, such as following KDEL targeted HRP transport through the ER network of transfected cells (Puhka et al., 2012). The advantage of HRP staining is that the reagents are readily available and are easy to use; however the DAB precipitation tends to spread from the point where the HRP is located and the stain may mask underlying features. A set of protein tags have been created, which are both fluorescent and able to generate an electron dense stain. In 2011 miniSOG (singlet oxygen generator) was shown to be able to fluoresce and that whilst doing so it could generate reactive oxygen species (Shu et al., 2011). The reactive oxygen 
species could then be used to precipitate DAB (Boassa et al., 2013). The only down side with the need for light activation was that the protein could not be localised by EM deeper than the fluorescence could be visualised. A different protein tag is an engineered ascorbate peroxidase (APEX), which was able to fluoresce and precipitate DAB for EM localisation (Martell et al., 2012). The peroxidase reaction works in a similar fashion to HRP, but the DAB appears to spread less producing a more specific localisation. The APEX molecule is being used in a variety of research to track labelled proteins by light microscopy and EM (Lee et al., 2016).

\subsection{Imaging the block face}

The processed samples may be surveyed in the microscope using the backscattered electron detector in order to locate target areas. Before surveying, the resin embedded samples are generally cut to around $1 \mathrm{~mm}$ and mounted on aluminium

pins. The samples can be smoothed using a conventional ultramicrotome and a diamond knife in order to optimise imaging, and the samples are often imaged at this point in order to assess tissue composition, preservation and stain quality. Once the face of the sample has been cut down to a suitable size (around $0.5 \times 0.5 \mathrm{~mm}$ for the automated imaging stage), the sample can be placed in the microscope and aligned to the knife, a process which involves ensuring that the knife will cut the sample as desired and bringing the sample block face to the cutting plane (this must be done with great care to avoid damage to the sample or the expensive diamond knife). The sample is mounted so that the freshly cut block face is pointing directly at the microscope pole piece. The imaging cycle involves scanning the electron beam across the surface of the block in order to generate an image with contrast derived from the signal of backscattered electrons which are deflected from the electron beam to a detector by the heavy-metal atoms bound to the biological material (figure 
4). During imaging the knife is parked off to one side so that it does not interfere with the data collection (figure $2 \mathrm{~A}-\mathrm{C}$ ). During the next step the knife is brought across between the sample and the microscope pole piece where the back scattered electron detector is mounted (figure D-F). In order to get the best image quality the shortest working distance (the distance between the sample and the detector) is needed, but this is restricted by the need to pass a knife between the detector and the sample. By precisely controlling the height of the sample during cutting it is possible to adjust the amount of the sample that is removed as the knife passes across the surface and specify the slice thickness and therefore the final Z-direction resolution of the resulting dataset. Once the section is cut the knife moves back to the safe position and a new image is collected. This cycle continues automatically with no need to refocus, as the sample working distance remains constant as this is set by the knife cutting position.

\subsection{Imaging and charging}

In order to take an image, the microscope needs to detect the signal strength at regularly-spaced physical points on the sample face which correspond to the pixels of a digital image. The signal strength is determined by the amount of backscattered electrons (BSE) that reach the BSE detector (figure 4). BSE are primary electrons from the main beam which interact with the sample and are then deflected back towards the detector, which sits below the pole piece of the microscope (Lloyd, 1987). Primary electrons which are incident on the sample surface interact with the atoms of the sample in nearly-elastic scattering events which can cause deflections in the angle of the primary electrons, resulting in some escaping the sample and being detected as BSE. The probability of a primary electron being back-scattered is increased by the presence of heavy metal atoms in the sample (Bozzola and 
Russell). Primary electrons are high energy electrons and the scattering events cause many lower energy secondary electrons (SE) to be produced which are also detectable and are used to generate typical biological SEM images. SE are electrons that originate from the sample and are knocked out of their orbitals by the primary electrons. SE are low energy and are quickly reabsorbed by atoms in the sample. As a result the only SE electrons that escape to form the image are those at the very top of the sample surface. BSE, however, are of a much higher energy and as a result can undergo many scattering events at a depth within the sample and still be detected (figure 4). Therefore BSE images give information about the atomic constitution of sample material that is below the surface. The depth of primary electron penetration and BSE detection is dependent on the energy of the electron beam and the constitution (atomic weight, density, crystal structure) of the sample. This means that the primary beam energy can be altered in order to ascertain information from different depths. Resultant images will contain overlapping information and therefore require computational deconvolution such as described in this paper (de Goede et al., 2017). This multi-energy deconvolution technology has been commercialised by Thermo Fischer in the form of the Teneo Volumescope ${ }^{\mathrm{TM}}$.

During imaging the electron beam is focused to a point that is smaller than the pixel size. The beam is left at this point for a number of microseconds (a period known as the dwell time) and the signal intensity is measured by the BSE detector. BSE detector signal intensity will become the pixel intensity at the first point in the image. After the dwell time has passed the scan coils then move the beam by 1 pixel length and the process is repeated as the beam is moved across the block face in a raster pattern, generating an image. The sample is cut by the knife at the specified slice thickness and the process repeats. During imaging secondary electrons may be generated at a faster rate than they are removed which can cause magnetic fields to 
be generated which act as localised lenses causing distortions in the images and severely reduce image quality. Secondary electrons can also contaminate the BSE detector signal if sufficient charge builds up. In a highly conductive sample (for example one with an abundance of well-stained material) the charge produced in the sample will travel to the earth via the stage and prevent charging artefacts. However with non-conductive samples (such as those with lots of empty resin, which is not conductive) more effort has to be used to remove the charge build up. The main method to reduce SE production is to reduce the primary beam power (current and voltage), reduce beam dwell time, or to add a charge reducing gas by increasing the chamber pressure in variable pressure systems (operating in 'low vacuum' mode). High vacuum imaging allows for higher resolution images but is rarely feasible due to charging build-up. Highly innervated tissue such as that of the brain and nerves can be imaged at high vacuum more easily than other tissue without any special preparations due to its relatively high inherent conductivity when stained with heavy metals (Deerinck et al., 2010a; Kornfeld et al., 2017; Pipkin et al., 2016; Sai et al., 2017).

The electron dose not only adversely affects imaging but also the cutting of the sample via the in-chamber microtome. The electrons moving around the sample as the beam is scanned across the surface can cause chemical bonds in the resin to become broken, which weakens the resin (Longiéras et al., 2006). This limits the total electrical dose that can be used to image a sample, as the weakened resin exhibits much poorer cutting properties and is prone to cutting problems. Typically, damaged resin is observed to the microscope operator as 'chatter' - where the knife repeatedly skips across the cutting surface destructively, reducing image quality. It may also be seen as 'cut and skip', wherein the resin is softened and is compressed by the knife rather than cut. With softened resin the sample may only be 
successfully cut once every several images. It is not uncommon to observe both these artefacts at separate points on an image stack, especially in samples with areas of varying conductivity.

Problems due to charging can limit image quality and have the potential to ruin entire datasets. As a result, research is ongoing into ways to reduce these issues. Research has examined the use of sample stage bias to reduce the beam-landing energies of the main beam electrons in SBFSEM. This technique has been known to improve SEM BSE imaging (Ohta et al., 2012). Bouwer et al. (Bouwer et al., 2017) observed that applying a negative sample stage bias reduced beam landing energies, reduced sample damage and increased BSE signal-to-noise ratio by accelerating BSE towards the BSE detector above the sample. In conjunction with a special BSE detector they were also able to alter the trajectories of primary and secondary electrons and control beam penetration depth. Artefacts such as drift and astigmatism were observed however the researchers believe that these can be addressed with more research.

Ohno and co-workers have experimented with creating conductive resins to decrease charging and had success with the conductive carbon black filler Ketjen black (Nguyen et al., 2016). They established that conductive resins reduce charging in the sample and also that reduced charging equates to better imaging and cutting. A difficulty we have noted when experimenting with Ketjen black at Manchester is that the conductive resin blocks are the same colour as the stained samples, which means that more care must be taken to identify the locations of samples during trimming and mounting. Silver has also been successfully deployed as a conductive resin component in a study which utilised SBFSEM to reconstruct a zebrafish olfactory bulb (Wanner et al., 2016). The authors observed that the 
sample exhibited high conductivity and were able to image a previously charging sample at high vacuum with $25 \mathrm{~nm}$ cut thickness.

Another approach to reducing charging is to add conductive material to the resin surface inside the microscope chamber during imaging and cutting cycles. Titze and Denk have experimented with fitting an electron beam evaporator into the SEM chamber (Titze and Denk, 2013) in order to coat the sample with conductive material between cuts. It was demonstrated that a 1-2nm film of palladium deposited onto the block between imaging rounds improved various aspects of imaging with only minor drawbacks. Another powerful technique detailed in a recent study features the creation of a small device that attaches to the $3 \mathrm{view}^{\mathrm{TM}}$ microtome and disperses nitrogen gas over the sample surface (Deerinck et al., 2018) in a method called Focal Charge Compensation. Secondary electrons created during imaging ionise the nitrogen molecules which then neutralise charging (L. et al., 1997). Targeting the gas at the block face allows the pressure in the rest of the chamber to be near to high vacuum, minimising the deflection of electrons in the beam by particles in the air. Focal Charge Compensation facilitated the imaging of previously difficult samples at near high vacuum and also allowed for high-resolution imaging of samples which had been stained in preparation for conventional TEM. This opens the door for SBFSEM to be performed on archival tissue bank samples that were originally prepared for TEM.

\subsection{Correlative SBFSEM}

The objective of correlative imaging is to allow for the location of the same region of interest (ROI) across different imaging modalities in order to gain information at different length scales. The concept of using correlative techniques in order to 
visualise features first by light microscopy and then by electron microscopy is being increasingly used with a serial block face approach (Armer et al., 2009; Knott et al., 2009; Puhka et al., 2012). In practice transferring between light microscopy and electron microscopy is often troublesome and there have been many different techniques developed over past decades. Correlative procedures for block face imaging techniques incorporate such methods as immunohistochemical staining (Sonomura et al., 2014), X-ray imaging (Bushong et al., 2015) and the use of fiducial markers near to the ROI (Maco et al., 2013). For cells in culture it is possible to grow the cells onto an etched coverslip to aid registration. The etching can be visualised in the light microscope and the same region can be found on the top of the resin block following embedding before trimming (Booth et al., 2013; Samejima et al., 2018). Fiducial markers can also be created whilst imaging using light microscopes via the use of high energies to create markers in the resin (Bishop et al., 2011). Near-infra red branding is based on the use of a two-photon microscope and was first used to correlate confocal imaging and SBFSEM in 2015 (Urwyler et al., 2015). The ROI is detected using the microscope before the beam energy of the laser is increased as to burn the sample within micrometers of the desired location. The edges of the burn are fluorescent and the mark can be located post-en-bloc staining as it will not take up any heavy metal stain. A detailed protocol for this method can be found here (Lees et al., 2017).

DAB can be utilised for SBFSEM such as in this (Ou et al., 2015) method wherein the DAB-oxidising protein MiniSOG is applied to a viral protein and the resulting DAB polymer can be produced via photo-oxidisation and viewed via SBFSEM following en-bloc staining. Attempts at correlative fluorescence and electron microscopy can be limited by the fact that EM staining procedures render samples opaque to light. Furthermore fluorescence is often lost during the EM staining and embedding 
process, however modified protocols have been developed that allow for their retention and subsequent imaging (Bell et al., 2013; Peddie et al., 2014). This allows for the imaging of TEM sections via fluorescence microscopy; however this process is difficult to transfer to the block face EM techniques as the sections are not preserved during imaging and cannot be retrieved. The Collinson group has developed a small fluorescence microscope that clips to the knife of the $3 v^{2} \mathrm{w}^{\mathrm{TM}}$ and fits inside the microscope chamber in order that light microscope images can be collected alongside the EM data as the sections are cut (Brama et al., 2016). This mini-microscope is designed to be retrofitted to SBFSEMs and facilitates the collection of fluorescence microscopy images in between imaging/cutting cycles. Xray microscopy is also applicable for correlative imaging, such as described in this paper (Bushong et al., 2015), wherein several methods are tested, such as the use of DAB photo oxidation to correlate markers in X-ray and SBFSEM images. A good place to learn more about the general concepts of CLEM would be (Muller-Reichert and Verkade, 2014).

\section{Image Analysis Procedures}

A benefit of the SBFSEM system is the ease with which it can generate large detailed datasets, but this also presents its own problem. Interpreting the data is far more complex than learning to recognise the individual organelles. Whilst complete datasets can be very visually impressive, for scientific purposes it is typically necessary to perform some form of data analysis before any results can be ascertained. Typically this data analysis comes in the form of segmentation - the process of assigning voxels to user-created structures - or in the form of quantitative measurements by a method such as stereology. There are several commercial and 
freeware software packages available that are commonly used for SBFSEM data processing and their capabilities are constantly improving, with an emphasis on developing automated methods of segmentation that make the process less time consuming. A good place to start learning about image analysis of EM volume data sets are (Cocks et al., 2018; Tsai et al., 2014), but here we will run through the general concepts.

\subsection{Segmentation}

Segmentation is the process of assigning pixels to objects, giving them a 'label'. In the case of SBFSEM the pixels are actually 3D voxels. Segmentation allows for the visualisation of important structures and interactions that lie within often complicated datasets. Segmentation also allows for quantitative measurements such as size, number and relative position to be performed. Presently, segmentation is performed both manually by trained scientists and automatically by computer algorithms that have varying degrees of human guidance. Despite ever-increasing amounts of computational aide, segmentation still has a deserved reputation as a mostly labourintensive and time consuming process.

Basic thresholding is segmentation at its simplest. A threshold value of voxel intensity is selected by the user and all voxels with an intensity above and equal to the intensity value are assigned to an object. This works particularly well when most of the sample has low stain content but a selective stain highlights the region of interest, such as lanthanum dysprosium staining of the glycosaminoglycan within the kidney glomeruli (Arkill et al., 2014), by staining individual neurons with biocytin (Lang et al., 2011), or where staining is low in t-tubules compared to the surrounding cardiac cells (Pinali et al., 2013). These approaches can be used to quickly gain an 
appreciation of the overall disorder within a volume of data (Pingel et al., 2014). The thresholded data can be used to generate models that can be interrogated, or it can be used for simple visualisation of the 3D arrangement of selected features.

Manual segmentation is any segmentation where a human user assigns pixels to objects individually. This often occurs in the form of tracing, with a mouse or touchsensitive screen used to 'paint' labels onto the 3D stack of images (an example of manual segmentation is shown in figure $1 \mathrm{C}$ where the chromosomes have been manually traced in IMOD (Kremer et al., 1996)). In many cases with a little training it is possible to segment most data with a reasonable degree of accuracy. Manual tracing can be tedious, but the time spent looking at the images often reveals detail that would previously have been hidden. This process can be made faster by techniques such as interpolation (where every nth image slice is segmented and a computer interpolation algorithm fills in the blank slices based on the manually segmented images) (Holcomb et al., 2013; Kornfeld et al., 2017). Manual segmentation can itself be computer-aided, with tools such as 'magic wand' brushes and superpixel-based tools such as those provided by software packages like TrakEM2 in Fiji (Cardona et al., 2010), MIB (Belevich et al., 2016) and SuRVoS (Luengo et al., 2017).

\subsection{Trainable algorithms}

Since manual segmentation often requires large amounts of human time and effort, and that it is a possible source of bias, bioimage analysts go to great lengths to try and automate segmentation as much as possible via the creation of image segmentation algorithms, which attempt to segment images based on user-defined or pre-existing criteria. Several algorithms exist which segment images based on 
information garnered by analysing images which have been manually segmented by humans. Fully automated segmentation is problematic and we are still at a level where it is generally not applicable for most purposes (this is not for want of trying; many groups put a lot of effort into exchange of ideas and training to improve image analysis procedures, such as (NEUBIAS)). However, progress is being made and it is not unreasonable to hope that in the coming years that algorithms will have the power to deal with a host of 3DEM image analysis requirements.

A recent paper demonstrates a MATLAB (MathWorks) program that will define cardiac cell structure based on rough segmentations performed by users at regular slice intervals (Hussain et al., 2018). Groups have experimented with tracing individual nerve fibres in a large dataset based on a single manually segmented slice (Kreshuk et al., 2015). By restricting the tracking to a specific question it is possible to generate algorithms that can be used to trace features through the volume, such as tracking the path of thousands of collagen fibirls in a volume of developing tendon (Almutairi et al., 2015). The users here were computer scientists who wrote specific software in order to work with this particular data set, something which a lot of scientists do not have the ability or resources to achieve. There are options for automated segmentation in the previously mentioned image segmentation programs, for example software such as MIB, using Simple Linear Iterative Clustering (Achanta et al., 2012) and Fiji using Weka (Arganda-Carreras et al., 2017) now offer opportunities for scientists to utilise less specific trainable algorithms. By defining a few voxels that belong to the object of interest along with a few voxels that are not the object of interest (often as simple lines drawn across the image) the software will find what is calculates to be analogous regions based on the user specification. By repeatedly checking the model produced and tweaking the user input the classifier generated by the algorithm can be refined and improved. The downside to this semi- 
automated approach is that in complex volumes it can be harder to check the accuracy of the selection, especially in the middle of the volume. The time taken to find the best filter for a particular data set and the potential need for different filters to pick out different organelles can put people off if they are used to getting immediate and accurate traces via manual segmentation. However the semi-automated methods work across larger volumes with each iteration and so the user will see larger trends more quickly than via the tracing of each section individually. Often the settings options for these programs, for example FIJI's WEKA segmentation plugin, rely on reasonably advanced mathematical terminology and lack explanation, making it difficult for those with a biological background to understand and use them.

The best method of training an automated segmentation algorithm is to train it with datasets that have been manually segmented by multiple users in order to minimise bias. However many smaller groups do not have resources to manually segment all of their own data sets, let alone segment data that has already been segmented so that software can find the few voxels that differ. A potential answer to this issue (as well as the issue of how to segment large volumes in general) is to use crowd sourcing, for example through scientific outreach approaches like 'citizen science' in the UK (Zooniverse). The issue now becomes one of how to train the user in order that you trust their tracing and how to retain the trained users by making the experience enjoyable. Groups have experimented with creating games where users fly through the volume (for example (Eyewire), or (Brainflight)), or making the task simple. Such as showing an image with 2 points in it and asking the user if they represent the same feature (Giuly et al., 2013). Most data produced in SBFSEM is used only to answer one or two biological questions and thorough analysis is not performed. As each dataset is rich with information that could be useful to users with other interests and for the training of algorithms. It has therefore been suggested 
that the public archiving of this data would be advantageous, allowing users to apply these algorithms to vast quantities of old data (Patwardhan et al., 2014).

\subsection{Stereology}

Stereology facilitates a more statistical approach to data analysis. A few recent papers have used SBFSEM to look at the relative volumetric proportions of organelles, for example comparing mitochondrial content in astrocytes and axons (Ju et al., 2015), or measuring synapse distribution along an axon (Salloum et al., 2014). They completed these measurements by manually tracing the volumes of interest and running the mathematics on the data produced, however a simpler method would have been to examine the data using a stereological approach, such as assessing the change in synapse density following prion infection (Peretti et al., 2015). The general concept is that by examining very small regions the data spread throughout the volume it is possible to gain quick estimates of organelle parameters. Lucocq et al estimate that to generate accurate estimates of organelle parameters "less than one thousandth" of the total volume of a 3D dataset needs to be examined

with a stereological approach (Lucocq et al., 2015). The mathematics can look daunting, but the concept is sound and, whilst it is always impressive to see beautiful 3D reconstructions, sometimes the question is better answered with statistical methods. For these sorts of questions, for example if a certain treatment has resulted in a cell having more of a certain organelle, a stereological approach makes sense as large data sets can be compared quickly and reproducibly. The ability to quickly analyse many datasets also allows for the quick identification of anomalous datasets and for the assessment of statistical trends, allowing more in-depth analysis to be saved for the datasets which best illustrate the scientific question being asked. 
A good place to start learning about stereology is a textbook such as this one (Howard and Reed, 2004).

\section{Future developments}

As has SBFSEM moved from a novel visualisation technology into a well-established tool for ultrastructural investigation, researchers have approached the limitations of the technique from all angles in order to improve its capabilities and practicality. Presently, SBFSEM stands as a technique suitable for the investigation of the 3D ultrastructure of cells and tissue across a wide range of sizes and constitutions. Researchers are expanding, pushing and refining the technique from all directions; imaging bigger things at higher resolutions, increasing the computational capability of image analysis tools and improving or inventing microscope components. Recent developments that reduce some of block face imaging's limitations, such as improved correlative capabilities, improved sample fixation and reduced charging, will mean that as these technologies are commercialised more powerful SBFSEM will be available to scientists around the world. It is not difficult to imagine that a combination of the new technologies discussed in this review will result in new SBFSEM microscopes with the capability to image bigger things in a shorter time at a higher resolution, and that the segmentation of the resulting data will be handled by an ever-more-powerful array of faithful computational helpers. In the coming years, SBFSEM has the potential to become one of the foremost experimental tools for the biological studies. 


\section{Acknowledgments}

The authors would like to thank Sarah Woolner (Wellcome Centre for Cell Matrix Research, Manchester) for the use of data to produce figure 1 and the sample used to generate images in figure 4. They also wish to thank Rene B. Svensson (Institute of Sports Medicine Copenhagen, Denmark), Klaus Qvortrup (Core Facility for Integrated Microscopy, Copenhagen, Denmark) and Karl Kadler (Wellcome Centre for Cell Matrix Research, Manchester, UK) for the use of the data to produce figure 3. They would also like to thank the staff of the Electron Microscope facility in the Faculty of Biology Medicine and Health, Manchester for their continued support. Finally they wish to thank the Wellcome Trust for funding. 


\section{Figure Legends}

Figure 1: SBFSEM: non-specific stains allow imaging of the whole system at high resolution. A) Confocal image of a dividing Xenopus epithelial cell. The $\alpha$ tubulin is labelled in green and the condensed chromatin is stained with DAPI (blue). The image only shows the location of the spindles and chromosomes B) Volume rendering of a dividing Xenopus epithelial cell imaged using SBFSEM. The condensed chromatin is rendered in blue. The two centrioles are just visible at the spindle poles coloured red. The rest of the cell is rendered based on stain density in white and pink. The volume rendering mainly shows the location of the pigment and lipids seen in the cytoplasm due to their electron dense staining. C) SBFSEM slice shown in B demonstrating how many more organelles can be seen due to the nonspecific staining. The condensed chromosomes are outlined in blue. The red square indicates the location of one of the centrioles. Note that this is a small (54 $\mathrm{x}$ $50 \mu \mathrm{m})$ part of a much larger $(84 \times 67 \times 142 \mu \mathrm{m})$ volume. D) A series of images centred in the same $x-y$ location as the red square in C. Each image is $100 \mathrm{~nm}$ deeper into the tissue than the image above it. The depth to the image compared with the slice shown in $\mathrm{C}$ is shown top right of each image. The data for this figure

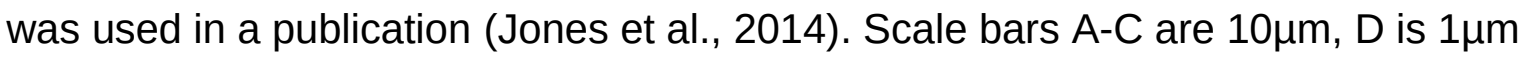

Figure 2 SBFSEM cutting cycle: Serial block face scanning electron microscopes utilise a small microtome that fits inside the scanning electron microscope with the sample held directly below the SEM pole piece. A and $\mathbf{D}$ show an image taken using the internal CCD mounted in our Quanta 250 FEG with the knife in the image position (A), or the cut position (D). The image has been annotated using (Inkscape) and the annotation is shown in B and E. The SBFSEM procedure builds up a three- 
dimensional data set by repeating a cycle of cutting followed by imaging of the newly exposed block face. In the first part of the cycle (C). The SEM is used to scan the beam across the surface of the sample. The back-scattered electrons that are generated by the interaction of the beam with atoms in the sample are collected using the BSED and an image is formed. Once an image has been collected, the knife holder passes between the back-scattered electron detector (BSED) and the sample (D). The sample is then raised a controlled amount and the diamond knife moves across the surface to take a thin (20-200nm) slice (F). Once the slice has been taken the knife holder is moved out of the way so that the cycle can repeat.

Figure 3 SBFSEM can be used to image large volumes at high resolution: Here a murine stapedius tendon was scanned from the myotendinous junction through the tendon to the stapes bone (A, C and D). The data set was collected with $15 \mathrm{~nm}$ pixels and 150nm cut size in order to track collagen fibrils through the entire length of the tendon. The collagen fibrils are highly aligned and elongated along the tendon long axis, so a lower resolution in $Z$ could be used to speed up the imaging process. The whole data stack took 7 days to collect with initial images covering $6000 \times 6000$ pixels $(90 \times 90 \mu \mathrm{m})$. Deeper into the sample the image size was reduced to $5000 \times 5000$ pixels $(75 \times 75 \mu \mathrm{m})$. The region scanned was re-centred a few times during the data collection in order to keep the tendon in the field of view. The overall volume shown in $\mathbf{E}$ is $125 \times 96 \times 370 \mu \mathrm{m}$. The resolution was sufficient that individual collagen fibrils can be seen in the area near to the myotendinous junction (see enlargement of the image $\mathbf{A}$ in $\mathbf{B})$. Amira was used to volume render the main regions of interest in order to show the overall volume imaged. The three regions rendered are muscle (red) tendon (blue) and bone (cream). The position of each example image (A, C and $\mathbf{D}$ ) is shown in the volume. Scale bars for the example images are $10 \mu \mathrm{m}$, apart from the enlargement (B), which is $500 \mathrm{~nm}$. The volume render scale bar is $25 \mu \mathrm{m}$, 
but due to the use of perspective in rendering the volume this should be taken as an approximation. This data set was generated in collaboration with users at CFIM, Copenhagen and was used in a publication (Svensson et al., 2017).

Figure 4: Primary Beam energy affects the depth of imaging: The backscattered electron image pixel intensity is predominantly affected by the density and atomic number of the atoms that the beam interacts with. Here we have used Monte Carlo simulation (Demers et al., 2011) to simulate the interaction of the beam at 1, 3 and $5 \mathrm{kV}$. The simulation of the beam interaction with empty epoxy resin (atomic fraction: 0.46 carbon, 0.444 hydrogen 0.096 oxygen) demonstrates how increasing the accelerating voltage increases the penetration of the beam within the sample. The depth of penetration in $100 \%$ Osmium, however, is much lower as the density is far higher and the higher atomic mass of the atoms causes larger deflections of the primary beam electrons. It can be seen that the path that each electron takes is apparently random with some interacting close to the surface, whiles others penetrate deeper and are eventually absorbed. The number of electrons being back-scattered and thus the pixel intensity is far higher in the osmium sample than the epoxy samples (in these simulations, back-scattering coefficient: $1 \mathrm{kV}$ epoxy= $0.087,1 \mathrm{kV}$ osmium $=0.343)$. In reality other factors will affect image quality, such as detector sensitively at lower accelerating voltage. The effect of the changes in beam penetration can be seen in the example inverted-contrast images. At low accelerating voltage the image is noisier due to lower detector sensitivity, but detail of the Golgi complex can be made out. As the accelerating voltage is increased the increased depth of the beam penetration reveals underlying membranous structures that were hidden in the low $\mathrm{kV}$ image (red arrows). Scale bars are $1 \mu \mathrm{m}$. 


\section{References}

Achanta, R., Shaji, A., Smith, K., Lucchi, A., Fua, P., Süsstrunk, S., 2012. SLIC superpixels compared to state-of-the-art superpixel methods. IEEE transactions on pattern analysis and machine intelligence 34, 2274-2282. Almutairi, Y., Cootes, T.F., Kadler, K., 2015. Tracking Collagen Fibres through Image Volumes from SBFSEM, MIUA, pp. 40-45.

Arganda-Carreras, I., Kaynig, V., Rueden, C., Eliceiri, K.W., Schindelin, J., Cardona, A., Sebastian Seung, H., 2017. Trainable Weka Segmentation: a machine learning tool for microscopy pixel classification. Bioinformatics 33, 2424-2426.

Arkill, K.P., Qvortrup, K., Starborg, T., Mantell, J.M., Knupp, C., Michel, C.C., Harper, S.J., Salmon, A.H., Squire, J.M., Bates, D.O., Neal, C.R., 2014. Resolution of the three dimensional structure of components of the glomerular filtration barrier. BMC Nephrol 15, 24.

Armer, H.E., Mariggi, G., Png, K.M., Genoud, C., Monteith, A.G., Bushby, A.J., Gerhardt, H., Collinson, L.M., 2009. Imaging transient blood vessel fusion events in zebrafish by correlative volume electron microscopy. PloS one 4, e7716.

Barcena, M., Koster, A.J., 2009. Electron tomography in life science. Semin Cell Dev Biol 20, 920-930.

Belevich, I., Joensuu, M., Kumar, D., Vihinen, H., Jokitalo, E., 2016. Microscopy Image Browser: A Platform for Segmentation and Analysis of Multidimensional Datasets. PLoS Biol 14, e1002340.

Bell, K., Mitchell, S., Paultre, D., Posch, M., Oparka, K., 2013. Correlative Imaging of Fluorescent Proteins in Resin-Embedded Plant Material(1). Plant Physiology 161, 1595-1603.

Biazik, J., Vihinen, H., Anwar, T., Jokitalo, E., Eskelinen, E.L., 2015. The versatile electron microscope: an ultrastructural overview of autophagy. Methods 75, 44-53.

Bishop, D., Nikic, I., Brinkoetter, M., Knecht, S., Potz, S.,

Kerschensteiner, M., Misgeld, T., 2011. Near-infrared branding efficiently correlates light and electron microscopy. Nat Methods 8, 568570 .

Boassa, D., Berlanga, M.L., Yang, M.A., Terada, M., Hu, J., Bushong, E.A., Hwang, M., Masliah, E., George, J.M., Ellisman, M.H., 2013. Mapping the subcellular distribution of alpha-synuclein in neurons using genetically encoded probes for correlated light and electron microscopy: implications for Parkinson's disease pathogenesis. J Neurosci 33, 26052615.

Booth, D.G., Cheeseman, L.P., Prior, I.A., Royle, S.J., 2013. Studying kinetochore-fiber ultrastructure using correlative light-electron microscopy. Methods Cell Biol 115, 327-342.

Bouwer, J.C., Deerinck, T.J., Bushong, E., Astakhov, V., Ramachandra, R., Peltier, S.T., Ellisman, M.H., 2017. Deceleration of probe beam by stage bias potential improves resolution of serial block-face scanning electron microscopic images. Adv Struct Chem Imaging 2, 11. 
Bozzola, J., Russell, L., Electron Microscopy. Principles and Techniques for Biologists 1999. Sudbury, Mass.[ua]: Jones and Bartlett. Brainflight, http://brainflight.org/. Date accessed: 31/05/18. Brama, E., Peddie, C.J., Wilkes, G., Gu, Y., Collinson, L.M., Jones, M.L., 2016. ultraLM and miniLM: Locator tools for smart tracking of fluorescent cells in correlative light and electron microscopy. Wellcome Open Res 1, 26.

Bushong, E.A., Johnson, D.D., Kim, K.-Y., Terada, M., Hatori, M., Peltier, S.T., Panda, S., Merkle, A., Ellisman, M.H., 2015. X-ray Microscopy as an Approach to Increasing Accuracy and Efficiency of Serial Block-face Imaging for Correlated Light and Electron Microscopy of Biological Specimens. Microscopy and microanalysis : the official journal of Microscopy Society of America, Microbeam Analysis Society, Microscopical Society of Canada 21, 231-238.

Cardona, A., Saalfeld, S., Arganda, I., Pereanu, W., Schindelin, J., Hartenstein, V., 2010. Identifying neuronal lineages of Drosophila by sequence analysis of axon tracts. J Neurosci 30, 7538-7553.

Chen, B., Yusuf, M., Hashimoto, T., Estandarte, A.K., Thompson, G., Robinson, I., 2017. Three-dimensional positioning and structure of chromosomes in a human prophase nucleus. Sci Adv 3, e1602231. Chuang, J.Z., Hsu, Y.C., Sung, C.H., 2015. Ultrastructural visualization of trans-ciliary rhodopsin cargoes in mammalian rods. Cilia $4,4$.

Cocks, E., Taggart, M., Rind, F.C., White, K., 2018. A guide to analysis and reconstruction of serial block face scanning electron microscopy data. J Microsc.

Daum, J.M., Keles, O., Holwerda, S.J., Kohler, H., Rijli, F.M., Stadler, M., Roska, B., 2017. The formation of the light-sensing compartment of cone photoreceptors coincides with a transcriptional switch. Elife 6.

de Goede, M., Johlin, E., Sciacca, B., Boughorbel, F., Garnett, E.C., 2017. 3D multi-energy deconvolution electron microscopy. Nanoscale 9, 684689.

Deerinck, T.J., Bushong, E., Lev-Ram, V., Shu, X., Tsien, R., Ellisman, M., 2010a. Enhancing Serial Block-Face Scanning Electron Microscopy to Enable High Resolution 3-D Nanohistology of Cells and Tissues. Deerinck, T.J., Bushong, E.A., Thor, A., Ellisman, M.H., 2010b. NCMIR methods for 3D EM: a new protocol for preparation of biological specimens for serial block face scanning electron microscopy. Microscopy, 6-8.

Deerinck, T.J., Shone, T.M., Bushong, E.A., Ramachandra, R., Peltier, S.T., Ellisman, M.H., 2018. High-performance serial block-face SEM of nonconductive biological samples enabled by focal gas injection-based charge compensation. J Microsc 270, 142-149.

Demers, H., Poirier-Demers, N., Couture, A.R., Joly, D., Guilmain, M., de Jonge, N., Drouin, D., 2011. Three-dimensional electron microscopy simulation with the CASINO Monte Carlo software. Scanning 33, 135146.

Denk, W., Horstmann, H., 2004. Serial block-face scanning electron microscopy to reconstruct three-dimensional tissue nanostructure. PLoS Biol 2, e329. 
Eyewire, https://eyewire.org/explore. Access date: 31/05/18.

Feeney, M., Kittelmann, M., Menassa, R., Hawes, C., Frigerio, L., 2018.

Protein Storage Vacuoles Originate from Remodeled Preexisting Vacuoles in Arabidopsis thaliana. Plant Physiology 177, 241-254.

Fiala, J.C., 2005. Reconstruct: a free editor for serial section microscopy. J Microsc 218, 52-61.

Fischer, S., Lu, Z., Meinertzhagen, I.A., 2018. From two to three dimensions: The importance of the third dimension for evaluating the limits to neuronal miniaturization in insects. J Comp Neurol 526, 653662.

Ghosh, S., Tran, K., M. D. Delbridge, L., Hickey, A., Hanssen, E., J. Crampin, E., Rajagopal, V., 2018. Insights on the impact of mitochondrial organisation on bioenergetics in high-resolution computational models of cardiac cell architecture.

Ghosh, S.K., 1981. Quantitative methods applied to stereo imaging. Theory. Methods Cell Biol 22, 155-176.

Giuly, R.J., Kim, K.Y., Ellisman, M.H., 2013. DP2: Distributed 3D image segmentation using micro-labor workforce. Bioinformatics 29, 13591360.

Glauert, A., 1984. Fixation, dehydration and embedding of biological specimens/Audrey M. Gauert. Practical methods in electron microscopy; v. 3 part 1.

Glauert, A.M., 1979. Recent advances of high voltage electron microscopy in biology. J Microsc 117, 93-101.

Godwin, A.R., Starborg, T., Sherratt, M.J., Roseman, A.M., Baldock, C., 2017. Defining the hierarchical organisation of collagen VI microfibrils at nanometre to micrometre length scales. Acta Biomater 52, 21-32. Hanker, J.S., 1979. Osmiophilic reagents in electronmicroscopic histocytochemistry. Prog Histochem Cytochem 12, 1-85.

Hayworth, K.J., Morgan, J.L., Schalek, R., Berger, D.R., Hildebrand, D.G., Lichtman, J.W., 2014. Imaging ATUM ultrathin section libraries with WaferMapper: a multi-scale approach to EM reconstruction of neural circuits. Front Neural Circuits 8, 68.

Holcomb, P.S., Hoffpauir, B.K., Hoyson, M.C., Jackson, D.R., Deerinck, T.J., Marrs, G.S., Dehoff, M., Wu, J., Ellisman, M.H., Spirou, G.A., 2013. Synaptic inputs compete during rapid formation of the calyx of Held: a new model system for neural development. J Neurosci 33, 12954-12969. Howard, V., Reed, M., 2004. Unbiased stereology: three-dimensional measurement in microscopy. Garland Science.

Hua, Y., Laserstein, P., Helmstaedter, M., 2015. Large-volume en-bloc staining for electron microscopy-based connectomics. Nat Commun 6, 7923.

Hughes, L., Borrett, S., Towers, K., Starborg, T., Vaughan, S., 2017. Patterns of organelle ontogeny through a cell cycle revealed by wholecell reconstructions using 3D electron microscopy. J Cell Sci 130, 637647.

Hussain, A., Ghosh, S., Kalkhoran, S.B., Hausenloy, D.J., Hanssen, E., Rajagopal, V., 2018. An automated workflow for segmenting single adult 
cardiac cells from large-volume serial block-face scanning electron microscopy data. J Struct Biol.

Inkscape, https://inkscape.org/en/. Date accessed: 31/05/18.

Jones, L.A., Villemant, C., Starborg, T., Salter, A., Goddard, G., Ruane, P., Woodman, P.G., Papalopulu, N., Woolner, S., Allan, V.J., 2014. Dynein light intermediate chains maintain spindle bipolarity by functioning in centriole cohesion. J Cell Biol 207, 499-516.

Ju, W.K., Kim, K.Y., Noh, Y.H., Hoshijima, M., Lukas, T.J., Ellisman, M.H., Weinreb, R.N., Perkins, G.A., 2015. Increased mitochondrial fission and volume density by blocking glutamate excitotoxicity protect glaucomatous optic nerve head astrocytes. Glia 63, 736-753.

Kaji, T., Kakui, K., Miyazaki, N., Murata, K., Palmer, A.R., 2016. Mesoscale morphology at nanoscale resolution: serial block-face scanning electron microscopy reveals fine 3D detail of a novel silk spinneret system in a tube-building tanaid crustacean. Front Zool 13, 14. Kalson, N.S., Starborg, T., Lu, Y., Mironov, A., Humphries, S.M., Holmes, D.F., Kadler, K.E., 2013. Nonmuscle myosin II powered transport of newly formed collagen fibrils at the plasma membrane. Proc Natl Acad Sci U S A 110, E4743-4752.

Kellenberger, E., Johansen, R., Maeder, M., Bohrmann, B., Stauffer, E., Villiger, W., 1992. Artefacts and morphological changes during chemical fixation. J Microsc 168, 181-201.

Khan, A.A., Riemersma, J.C., Booij, H.L., 1961. The reactions of osmium tetroxide with lipids and other compounds. J Histochem Cytochem 9, 560563.

Kittelmann, M., Hawes, C., Hughes, L., 2016. Serial block face scanning electron microscopy and the reconstruction of plant cell membrane systems. J Microsc 263, 200-211.

Knott, G., Marchman, H., Wall, D., Lich, B., 2008. Serial section scanning electron microscopy of adult brain tissue using focused ion beam milling. J Neurosci 28, 2959-2964.

Knott, G.W., Holtmaat, A., Trachtenberg, J.T., Svoboda, K., Welker, E., 2009. A protocol for preparing GFP-labeled neurons previously imaged in vivo and in slice preparations for light and electron microscopic analysis. Nat Protoc 4, 1145-1156.

Korkmaz, E., 2016. New Approach to 3-D Imaging of Biological Materials Provides Isotropic Resolution Down to a Few Nanometers. AMERICAN LABORATORY 48, 14-17.

Kornfeld, J., Benezra, S.E., Narayanan, R.T., Svara, F., Egger, R., Oberlaender, M., Denk, W., Long, M.A., 2017. EM connectomics reveals axonal target variation in a sequence-generating network. Elife 6. Kremer, J.R., Mastronarde, D.N., McIntosh, J.R., 1996. Computer visualization of three-dimensional image data using IMOD. J Struct Biol $116,71-76$.

Kreshuk, A., Walecki, R., Koethe, U., Gierthmuehlen, M., Plachta, D., Genoud, C., Haastert-Talini, K., Hamprecht, F.A., 2015. Automated tracing of myelinated axons and detection of the nodes of Ranvier in serial images of peripheral nerves. J Microsc 259, 143-154. 
L., T.B., C., B.I., L., F.A., P., M., M., D.A., 1997. An improved model for gaseous amplification in the environmental SEM. Journal of Microscopy 187, 143-157.

Lang, S., Drouvelis, P., Tafaj, E., Bastian, P., Sakmann, B., 2011. Fast extraction of neuron morphologies from large-scale SBFSEM image stacks. Journal of computational neuroscience 31, 533-545.

Lausecker, F., Tian, X., Inoue, K., Wang, Z., Pedigo, C.E., Hassan, H., Liu, C., Zimmer, M., Jinno, S., Huckle, A.L., Hamidi, H., Ross, R.S., Zent, R., Ballestrem, C., Lennon, R., Ishibe, S., 2018. Vinculin is required to maintain glomerular barrier integrity. Kidney International 93, 643-655. Lee, S.Y., Kang, M.G., Park, J.S., Lee, G., Ting, A.Y., Rhee, H.W., 2016. APEX Fingerprinting Reveals the Subcellular Localization of Proteins of Interest. Cell Rep 15, 1837-1847.

Lees, R.M., Peddie, C.J., Collinson, L.M., Ashby, M.C., Verkade, P., 2017. Chapter 12 - Correlative two-photon and serial block face scanning electron microscopy in neuronal tissue using 3D near-infrared branding maps, in: Müller-Reichert, T., Verkade, P. (Eds.), Methods in Cell Biology. Academic Press, pp. 245-276.

Leighton, S.B., 1981. SEM images of block faces, cut by a miniature microtome within the SEM - a technical note. Scan Electron Microsc, 7376.

Lloyd, G., 1987. Atomic Number and Crystallographic Contrast Images with the SEM: A Review of Backscattered Electron Techniques.

Longiéras, N., Sebban, M., Palmas, P., Rivaton, A., Gardette, J.-L., 2006. Multiscale approach to investigate the radiochemical degradation of epoxy resins under high-energy electron-beam irradiation. Journal of Polymer Science Part A: Polymer Chemistry 44, 865-887.

Lucocq, J.M., Mayhew, T.M., Schwab, Y., Steyer, A.M., Hacker, C., 2015. Systems biology in 3D space--enter the morphome. Trends Cell Biol 25, $59-64$.

Luengo, I., Darrow, M.C., Spink, M.C., Sun, Y., Dai, W., He, C.Y., Chiu, W., Pridmore, T., Ashton, A.W., Duke, E.M.H., Basham, M., French, A.P., 2017. SuRVoS: Super-Region Volume Segmentation workbench. J Struct Biol 198, 43-53.

Maco, B., Holtmaat, A., Cantoni, M., Kreshuk, A., Straehle, C.N., Hamprecht, F.A., Knott, G.W., 2013. Correlative In Vivo 2 Photon and Focused Ion Beam Scanning Electron Microscopy of Cortical Neurons. PloS one 8, e57405.

Martell, J.D., Deerinck, T.J., Sancak, Y., Poulos, T.L., Mootha, V.K., Sosinsky, G.E., Ellisman, M.H., Ting, A.Y., 2012. Engineered ascorbate peroxidase as a genetically encoded reporter for electron microscopy. Nat Biotechnol 30, 1143-1148.

Mazzone, R.W., Kornblau, S., Durand, C.M., 1980. Shrinkage of lung after chemical fixation for analysis of pulmonary structure-function relations. J Appl Physiol Respir Environ Exerc Physiol 48, 382-385. Micheva, K.D., Smith, S.J., 2007. Array tomography: a new tool for imaging the molecular architecture and ultrastructure of neural circuits. Neuron 55, 25-36. 
Mikula, S., Denk, W., 2015. High-resolution whole-brain staining for electron microscopic circuit reconstruction. Nat Methods 12, 541-546. Muller-Reichert, T., Verkade, P., 2014. Correlative light and electron microscopy II. Academic Press.

NEUBIAS, http://eubias.org/NEUBIAS/. Date accessed: 31/05/18. Nguyen, H.B., Thai, T.Q., Saitoh, S., Wu, B., Saitoh, Y., Shimo, S., Fujitani, H., Otobe, H., Ohno, N., 2016. Conductive resins improve charging and resolution of acquired images in electron microscopic volume imaging. Sci Rep 6, 23721.

Nixon, F.M., Honnor, T.R., Clarke, N.I., Starling, G.P., Beckett, A.J., Johansen, A.M., Brettschneider, J.A., Prior, I.A., Royle, S.J., 2017.

Microtubule organization within mitotic spindles revealed by serial block face scanning electron microscopy and image analysis. J Cell Sci 130, $1845-1855$.

Odriozola, A., Llodrá, J., Radecke, J., Ruegsegger, C., Tschanz, S., Saxena, S., Rohr, S., Zuber, B., 2017. High contrast staining for serial block face scanning electron microscopy without uranyl acetate. bioRxiv, 207472.

Ohta, K., Sadayama, S., Togo, A., Higashi, R., Tanoue, R., Nakamura, K., 2012. Beam deceleration for block-face scanning electron microscopy of embedded biological tissue. Micron (Oxford, England : 1993) 43, 612620 .

Ou, H.D., Deerinck, T.J., Bushong, E., Ellisman, M.H., O’Shea, C.C., 2015. Visualizing Viral Protein Structures in Cells Using Genetic Probes for Correlated Light and Electron Microscopy. Methods (San Diego, Calif.) 90, 39-48.

Parkyn Schneider, M., Liu, B., Glock, P., Suttie, A., McHugh, E., Andrew, D., Batinovic, S., Williamson, N., Hanssen, E., McMillan, P., Hliscs, M., Tilley, L., Dixon, M.W.A., 2017. Disrupting assembly of the inner membrane complex blocks Plasmodium falciparum sexual stage development. PLoS Pathog 13, e1006659.

Patwardhan, A., Ashton, A., Brandt, R., Butcher, S., Carzaniga, R., Chiu, W., Collinson, L., Doux, P., Duke, E., Ellisman, M.H., Franken, E., Grunewald, K., Heriche, J.K., Koster, A., Kuhlbrandt, W., Lagerstedt, I., Larabell, C., Lawson, C.L., Saibil, H.R., Sanz-Garcia, E., Subramaniam, S., Verkade, P., Swedlow, J.R., Kleywegt, G.J., 2014. A 3D cellular context for the macromolecular world. Nat Struct Mol Biol 21, 841-845.

Peddie, C.J., Blight, K., Wilson, E., Melia, C., Marrison, J., Carzaniga, R., Domart, M.-C., O'Toole, P., Larijani, B., Collinson, L.M., 2014. Correlative and integrated light and electron microscopy of in-resin GFP

fluorescence, used to localise diacylglycerol in mammalian cells.

Ultramicroscopy 143, 3-14.

Peddie, C.J., Collinson, L.M., 2014. Exploring the third dimension: volume electron microscopy comes of age. Micron (Oxford, England : 1993) 61, 9-19.

Pedler, C., Tilly, R., 1966. A new method of serial reconstruction from electron micrographs. Journal of Microscopy 86, 189-197.

Peretti, D., Bastide, A., Radford, H., Verity, N., Molloy, C., Martin, M.G., Moreno, J.A., Steinert, J.R., Smith, T., Dinsdale, D., Willis, A.E., Mallucci, 
G.R., 2015. RBM3 mediates structural plasticity and protective effects of cooling in neurodegeneration. Nature 518, 236-239.

Pinali, C., Bennett, H., Davenport, J.B., Trafford, A.W., Kitmitto, A., 2013. Three-dimensional reconstruction of cardiac sarcoplasmic reticulum reveals a continuous network linking transverse-tubules: this organization is perturbed in heart failure. Circ Res 113, 1219-1230. Pingel, J., Lu, Y., Starborg, T., Fredberg, U., Langberg, H., Nedergaard, A., Weis, M., Eyre, D., Kjaer, M., Kadler, K.E., 2014. 3-D ultrastructure and collagen composition of healthy and overloaded human tendon: evidence of tenocyte and matrix buckling. Journal of anatomy 224, 548555.

Pipkin, J.E., Bushong, E.A., Ellisman, M.H., Kristan, W.B., Jr., 2016. Patterns and distribution of presynaptic and postsynaptic elements within serial electron microscopic reconstructions of neuronal arbors from the medicinal leech Hirudo verbana. J Comp Neurol 524, 36773695.

Plachno, B.J., Swiatek, P., Jobson, R.W., Malota, K., Brutkowski, W., 2017. Serial block face SEM visualization of unusual plant nuclear tubular extensions in a carnivorous plant (Utricularia, Lentibulariaceae). Ann Bot 120, 673-680.

Pollreisz, A., Messinger, J.D., Sloan, K.R., Mittermueller, T.J., Weinhandl, A.S., Benson, E.K., Kidd, G.J., Schmidt-Erfurth, U., Curcio, C.A., 2018. Visualizing melanosomes, lipofuscin, and melanolipofuscin in human retinal pigment epithelium using serial block face scanning electron microscopy. Exp Eye Res 166, 131-139.

Puhka, M., Joensuu, M., Vihinen, H., Belevich, I., Jokitalo, E., 2012. Progressive sheet-to-tubule transformation is a general mechanism for endoplasmic reticulum partitioning in dividing mammalian cells. Molecular biology of the cell 23, 2424-2432.

Reynolds, E.S., 1963. The use of lead citrate at high pH as an electronopaque stain in electron microscopy. J Cell Biol 17, 208-212.

Rieder, C.L., 1981. Thick and thin serial sectioning for the threedimensional reconstruction of biological ultrastructure. Methods Cell Biol 22, 215-249.

Robinson, J.M., Takizawa, T., Vandre, D.D., 2000. Applications of gold cluster compounds in immunocytochemistry and correlative microscopy: comparison with colloidal gold. J Microsc 199, 163-179.

Rouquette, J., Genoud, C., Vazquez-Nin, G.H., Kraus, B., Cremer, T., Fakan, S., 2009. Revealing the high-resolution three-dimensional network of chromatin and interchromatin space: A novel electron-microscopic approach to reconstructing nuclear architecture. Chromosome Research $17,801$.

Saga, K., 2005. Application of cryofixation and cryoultramicrotomy for biological electron microscopy. Med Mol Morphol 38, 155-160. Sai, K., Wang, S., Kaito, A., Fujiwara, T., Maruo, T., Itoh, Y., Miyata, M., Sakakibara, S., Miyazaki, N., Murata, K., Yamaguchi, Y., Haruta, T., Nishioka, H., Motojima, Y., Komura, M., Kimura, K., Mandai, K., Takai, Y., Mizoguchi, A., 2017. Multiple roles of afadin in the ultrastructural 
morphogenesis of mouse hippocampal mossy fiber synapses. J Comp Neurol 525, 2719-2734.

Salloum, R.H., Chen, G., Velet, L., Manzoor, N.F., Elkin, R., Kidd, G.J., Coughlin, J., Yurosko, C., Bou-Anak, S., Azadi, S., Gohlsch, S., Schneider, H., Kaltenbach, J.A., 2014. Mapping and morphometric analysis of synapses and spines on fusiform cells in the dorsal cochlear nucleus. Front Syst Neurosci 8, 167.

Salo, R.A., Belevich, I., Manninen, E., Jokitalo, E., Grohn, O., Sierra, A., 2018. Quantification of anisotropy and orientation in 3D electron microscopy and diffusion tensor imaging in injured rat brain.

Neuroimage 172, 404-414.

Samejima, K., Booth, D.G., Ogawa, H., Paulson, J.R., Xie, L., Watson, C.A., Platani, M., Kanemaki, M.T., Earnshaw, W.C., 2018. Functional analysis after rapid degradation of condensins and 3D-EM reveals chromatin volume is uncoupled from chromosome architecture in mitosis. J Cell Sci 131.

Schalek, R., Kasthuri, N., Hayworth, K., Berger, D., Tapia, J., Morgan, J., Turaga, S., Fagerholm, E., Seung, H., Lichtman, J., 2011. Development of high-throughput, high-resolution 3D reconstruction of large-volume biological tissue using automated tape collection ultramicrotomy and scanning electron microscopy. Microscopy and Microanalysis 17, 966. Schultz, R.L., Maynard, E.A., Pease, D.C., 1957. Electron microscopy of neurons and neuroglia of cerebral cortex and corpus callosum. Am J Anat 100, 369-407.

Seligman, A.M., Wasserkrug, H.L., Hanker, J.S., 1966. A new staining method (OTO) for enhancing contrast of lipid--containing membranes and droplets in osmium tetroxide--fixed tissue with osmiophilic thiocarbohydrazide(TCH). J Cell Biol 30, 424-432.

Shu, X., Lev-Ram, V., Deerinck, T.J., Qi, Y., Ramko, E.B., Davidson, M.W., Jin, Y., Ellisman, M.H., Tsien, R.Y., 2011. A genetically encoded tag for correlated light and electron microscopy of intact cells, tissues, and organisms. PLoS Biol 9, e1001041.

Sonomura, T., Furuta, T., Nakatani, I., Yamamoto, Y., Honma, S., Kaneko, T., 2014. Attempt of correlative observation of morphological synaptic connectivity by combining confocal laser-scanning microscope and FIBSEM for immunohistochemical staining technique. Microscopy (Oxf) 63 Suppl 1, i8.

Soto, G.E., Young, S.J., Martone, M.E., Deerinck, T.J., Lamont, S., Carragher, B.O., Hama, K., Ellisman, M.H., 1994. Serial section electron tomography: a method for three-dimensional reconstruction of large structures. Neuroimage 1, 230-243.

Starborg, T., Kalson, N.S., Lu, Y., Mironov, A., Cootes, T.F., Holmes, D.F., Kadler, K.E., 2013. Using transmission electron microscopy and 3View to determine collagen fibril size and three-dimensional organization. Nat Protoc 8, 1433-1448.

Svensson, R.B., Herchenhan, A., Starborg, T., Larsen, M., Kadler, K.E., Qvortrup, K., Magnusson, S.P., 2017. Evidence of structurally continuous collagen fibrils in tendons. Acta Biomater 50, 293-301. 
Szczesny, P.J., Walther, P., Muller, M., 1996. Light damage in rod outer segments: the effects of fixation on ultrastructural alterations. Curr Eye Res 15, 807-814.

Testillano, P.S., Sanchez-Pina, M.A., Olmedilla, A., Ollacarizqueta, M.A., Tandler, C.J., Risueno, M.C., 1991. A specific ultrastructural method to reveal DNA: the NAMA-Ur. J Histochem Cytochem 39, 1427-1438.

Titze, B., Denk, W., 2013. Automated in-chamber specimen coating for serial block-face electron microscopy. J Microsc 250, 101-110.

Tsai, W.T., Hassan, A., Sarkar, P., Correa, J., Metlagel, Z., Jorgens, D.M., Auer, M., 2014. From voxels to knowledge: a practical guide to the segmentation of complex electron microscopy 3D-data. J Vis Exp, e51673. Tsang, T.K., Bushong, E.A., Boassa, D., Hu, J., Romoli, B., Phan, S., Dulcis, D., Su, C.Y., Ellisman, M.H., 2018. High-quality ultrastructural preservation using cryofixation for 3D electron microscopy of genetically labeled tissues. Elife 7.

Tsukamoto, Y., Omi, N., 2017. Classification of Mouse Retinal Bipolar Cells: Type-Specific Connectivity with Special Reference to Rod-Driven AII Amacrine Pathways. Frontiers in Neuroanatomy 11, 92.

Turner, J.N., 1981. Introduction to stereo imaging. Methods Cell Biol 22, $1-11$.

Urwyler, O., Izadifar, A., Dascenco, D., Petrovic, M., He, H., Ayaz, D., Kremer, A., Lippens, S., Baatsen, P., Guérin, C.J., Schmucker, D., 2015. Investigating CNS synaptogenesis at single-synapse resolution by combining reverse genetics with correlative light and electron microscopy. Development 142, 394-405.

Vincent, A.E., Ng, Y.S., White, K., Davey, T., Mannella, C., Falkous, G., Feeney, C., Schaefer, A.M., McFarland, R., Gorman, G.S., Taylor, R.W., Turnbull, D.M., Picard, M., 2016. The Spectrum of Mitochondrial Ultrastructural Defects in Mitochondrial Myopathy. Sci Rep 6, 30610. Volkert, C.A., Minor, A.M., 2007. Focused ion beam microscopy and micromachining. MRS bulletin 32, 389-399.

Wacker, I.U., Veith, L., Spomer, W., Hofmann, A., Thaler, M., Hillmer, S., Gengenbach, U., Schroder, R.R., 2018. Multimodal Hierarchical Imaging of Serial Sections for Finding Specific Cellular Targets within Large Volumes. J Vis Exp.

Wang, L., Hiler, D., Xu, B., AlDiri, I., Chen, X., Zhou, X., Griffiths, L., Valentine, M., Shirinifard, A., Sablauer, A., Thiagarajan, S., Barabas, M.E., Zhang, J., Johnson, D., Frase, S., Dyer, M.A., 2018. Retinal Cell Type DNA Methylation and Histone Modifications Predict Reprogramming Efficiency and Retinogenesis in 3D Organoid Cultures. Cell Reports 22, 2601-2614.

Wanner, A.A., Genoud, C., Friedrich, R.W., 2016. 3-dimensional electron microscopic imaging of the zebrafish olfactory bulb and dense reconstruction of neurons. Sci Data 3, 160100. Webb, R., Webb, R., 2015. Quick Freeze Substitution Processing of Biological Samples for Serial Block-face Scanning Electron Microscopy. Microscopy and Microanalysis 21, 1115-1116. 
Webb, R.I., Schieber, N.L., 2018. Volume Scanning Electron Microscopy: Serial Block-Face Scanning Electron Microscopy Focussed Ion Beam Scanning Electron Microscopy, Cellular Imaging. Springer, pp. 117-148. Willingham, M.C., Rutherford, A.V., 1984. The use of osmiumthiocarbohydrazide-osmium (OTO) and ferrocyanide-reduced osmium methods to enhance membrane contrast and preservation in cultured cells. J Histochem Cytochem 32, 455-460.

$\mathrm{Xu}$, C.S., Hayworth, K.J., Lu, Z., Grob, P., Hassan, A.M., Garcia-Cerdan, J.G., Niyogi, K.K., Nogales, E., Weinberg, R.J., Hess, H.F., 2017. Enhanced FIB-SEM systems for large-volume 3D imaging. Elife 6. Zooniverse, https://www.zooniverse.org/projects/h-spiers/etch-a-cell/about/research. Date accessed: 31/05/18. 


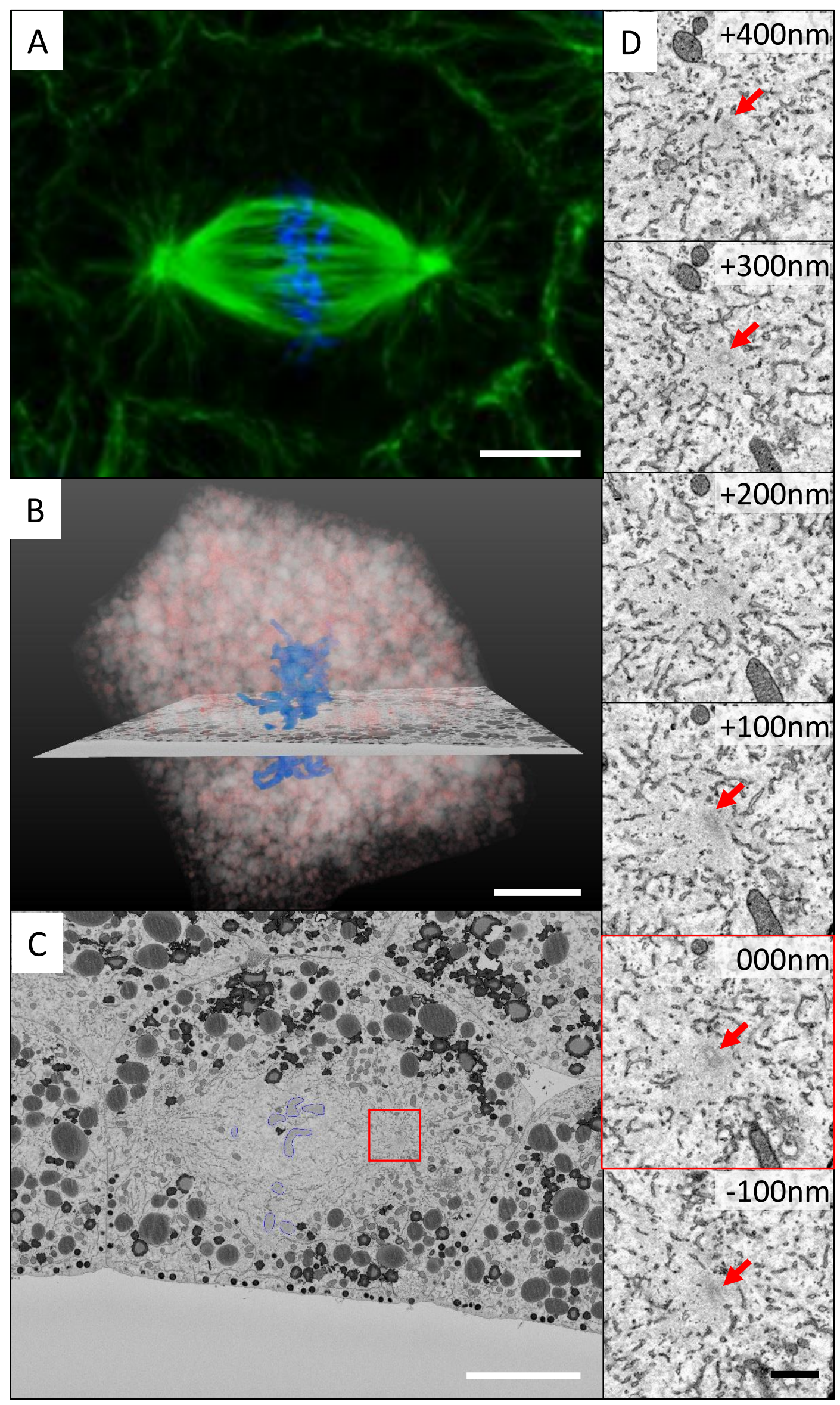




\section{Smith and Starborg Figure 2}

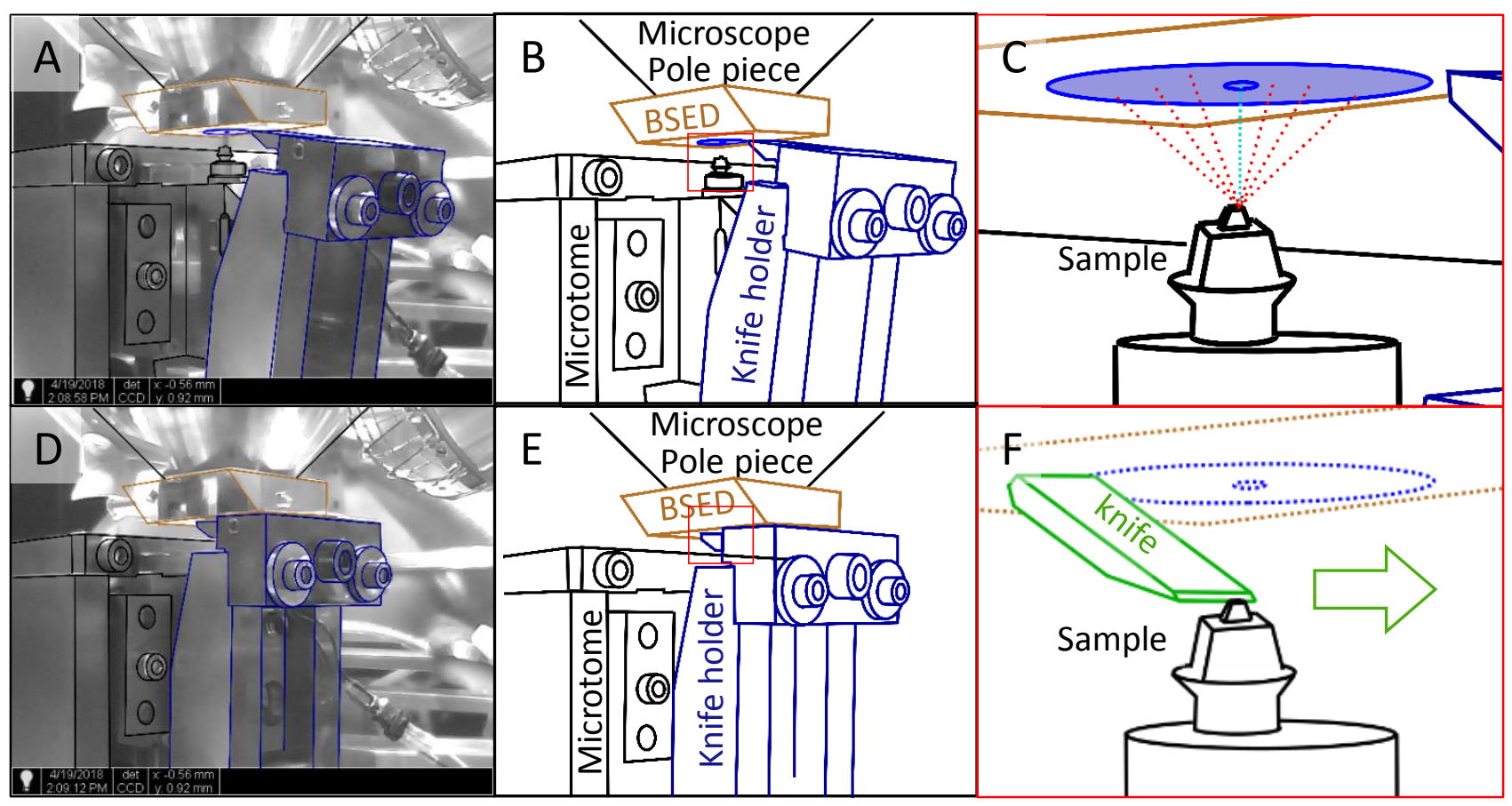




\section{Smith and Starborg Figure 3}

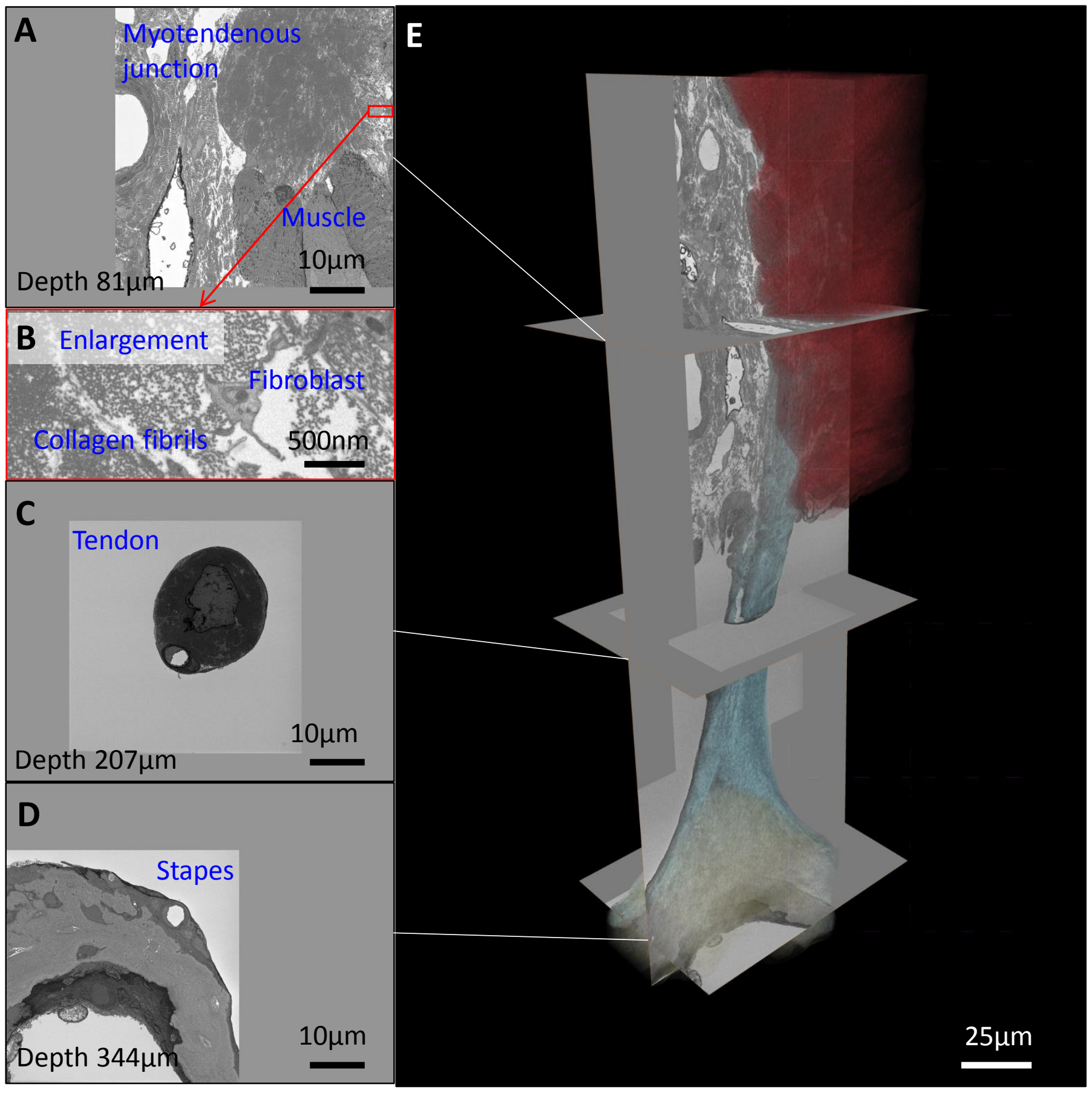


